\title{
Study of melanin and anthocyanin biosynthesis regulation in barley grain by transcriptomic analysis of near-isogenic lines with different pigment composition
}

\author{
Vikhorev A.V. ${ }^{1,2 *}$, Glagoleva A.Yu. ${ }^{1}$, Shmakov N.A. ${ }^{1}$, Kukoeva T.V. ${ }^{1}$, \\ Khlestkina E.K. ${ }^{1,3}$, Shoeva O.Yu. ${ }^{1}$ \\ ${ }^{1}$ Institute of Cytology and Genetics, SB RAS, Novosibirsk, Russia \\ ${ }^{2}$ Novosibirsk State University, Novosibirsk, Russia \\ ${ }^{3}$ N.I. Vavilov Research Institute of Plant Industry, St. Petersburg, Russia \\ *email:vikhorev@bionet.nsc.ru
}

Dark pigmentation of barley spike (Hordeum vulgare L.) can be caused by two types of phenolic compounds: anthocyanins that belong to flavonoids and provide blue or purple color of grain and black or brown melanins that are result of enzymatic oxidation of phenolic compounds. Different genes control the accumulation of these pigments: the complementary genes Ant 1 and Ant 2 determine synthesis of purple anthocyanins in grain pericarp; while melanins are formed under control of the Blp gene in hulls and pericarp tissues. These colorants can be accumulated in grain envelopes independently or simultaneously, and presumably the same phenolic substrates can be involved. However, the exact mechanisms of anthocyanins and melanin biosynthesis pathways interaction were not investigated previously. Using the marker-assisted selection the hybrid nearisogenic line (NIL) i:BwBlpAnt1Ant2, which simultaneously accumulates anthocyanins and melanin in grain was developed. In addition to parental black-grained i:BwBlp and purple-grained i:BwAnt1Ant2 NILs and initial cultivar Bowman the obtained line is a precise genetic model for comparative transcriptomic analysis. Total RNA from NILs at three stages of spike development (booting, late milk and early dough stages) was extracted and sequenced. In total, 36 sequenced Illumina mRNA libraries were obtained. We have found upregulation of the key flavonoid biosynthesis genes in hybrid line that occurs earlier than in the purple-grained line, at the late milk stage of spike maturation. Besides, the obtained transcriptomic data demonstrated the downregulation of photosynthesis genes in NIL accumulating melanin. Thus, we assume that the presence of the Blp locus increase anthocyanins biosynthesis and suppress chlorophyll biosynthesis in barley grain.

Acknowledgements: The study was supported by the RFBR grant No. 20-316-80016. 